Bond University

Research Repository

\title{
Effectiveness and sustainability of deprescribing for hospitalized older patients near end of life: a systematic review
}

Cardona, Magnolia; Stehlik, Paulina; Fawzy, Peter; Byambasuren, Oyungerel; Anderson, Jarrah; Clark, Justin; Sun, Shelley; Scott, lan

Published in:

Expert Opinion on Drug Safety

DOI:

10.1080/14740338.2021.1853704

Licence:

Other

Link to output in Bond University research repository.

Recommended citation(APA):

Cardona, M., Stehlik, P., Fawzy, P., Byambasuren, O., Anderson, J., Clark, J., Sun, S., \& Scott, I. (2021).

Effectiveness and sustainability of deprescribing for hospitalized older patients near end of life: a systematic review. Expert Opinion on Drug Safety, 20(1), 81-91. https://doi.org/10.1080/14740338.2021.1853704

\section{General rights}

Copyright and moral rights for the publications made accessible in the public portal are retained by the authors and/or other copyright owners and it is a condition of accessing publications that users recognise and abide by the legal requirements associated with these rights.

For more information, or if you believe that this document breaches copyright, please contact the Bond University research repository coordinator. 


\section{Effectiveness and sustainability of deprescribing for hospitalised older patients near end of life: $a$ systematic review}

\section{Magnolia Cardona , Paulina Stehlik, Peter Fawzy , Oyungerel Byambasuren, Jarrah Anderson, Justin Clark, Shelley Sun \& Ian Scott}

To cite this article: Magnolia Cardona, Paulina Stehlik, Peter Fawzy, Oyungerel Byambasuren, Jarrah Anderson , Justin Clark, Shelley Sun \& Ian Scott (2020): Effectiveness and sustainability of deprescribing for hospitalised older patients near end of life: a systematic review, Expert Opinion on Drug Safety, DOI: 10.1080/14740338.2021.1853704

To link to this article: https://doi.org/10.1080/14740338.2021.1853704

Accepted author version posted online: 19 Nov 2020.

Submit your article to this journal ¿

山ll Article views: 5

Q View related articles ¿

View Crossmark data $₫$ 
Publisher: Taylor \& Francis \& Informa UK Limited, trading as Taylor \& Francis Group Journal: Expert Opinion on Drug Safety

DOI: $10.1080 / 14740338.2021 .1853704$

\section{Effectiveness and sustainability of deprescribing for hospitalised older patients near end of life: a systematic review}

Magnolia Cardona, ${ }^{1,2}$ Paulina Stehlik, ${ }^{1,2}$ Peter Fawzy, ${ }^{1}$ Oyungerel Byambasuren, ${ }^{2}$ Jarrah Anderson, ${ }^{3}$ Justin Clark, ${ }^{2}$ Shelley Sun, ${ }^{4}$ Ian Scott ${ }^{5}$

1 Gold Coast University Hospital, Southport, QLD, Australia

2 Institute for Evidence-Based HealthCare, Bond University, Gold Coast, QLD, Australia

3 School of Pharmacy and Pharmacology, Griffith University, Gold Coast, QLD, Australia

$4 \quad$ Sydney Medical School, The University of New South Wales, Kensington, NSW, Australia

5 Princess Alexandra Hospital, Metro South, QLD Health, Brisbane, QLD, Australia

\section{Corresponding author:}

Magnolia Cardona,

Gold Coast University Hospital, Southport, QLD, Australia

Institute for Evidence-Based HealthCare, Bond University, Gold Coast, QLD, Australia

Email: mcardona@bond.edu.au 


\begin{abstract}
Introduction: Polypharmacy is prevalent in hospitals and deprescribing strategies for older people are strongly promoted. However, evidence of their feasibility and sustainability among patients receiving end of life care is lacking. The objective of this review was to ascertain effectiveness and post-discharge sustainability of hospital-initiated deprescribing strategies in older people near the end of life.

Areas covered. The authors searched for controlled trials, with low risk of bias and measures of effectiveness post-discharge. Intervention description, duration and healthcare provider engagement were investigated for their impact on reduction of number of medications, proportions of patients prescribed inappropriate medications, returns to emergency, hospital admission and adverse events.

Expert opinion. Limited evidence suggests hospital-initiated deprescribing interventions may reduce prescribing inappropriateness among older terminal patients in the short term, but evidence beyond 3 months is lacking for significant prevention of adverse events or health service utilisation. Heterogeneity precluded meta-analysis, and short follow-up periods precluded quantitative assessment of sustainability. Trials of older people with terminal conditions with larger sample sizes and longer follow-up periods are needed to confirm the effectiveness and sustainability of deprescribing at the end of life. Objective tools to reliably identify near end-of-life status would be useful in selecting target groups for these interventions.
\end{abstract}

Key words: aged; controlled trials; deprescribing; end-of-life; polypharmacy

Protocol registration: https://osf.io/9mv8d/ 


\section{Article highlights}

- Older people with progressive multiple comorbidities who are nearing the end of their lives often take multiple non-essential medicines which can impair their cognitive and physical functioning, and are potentially harmful. Medication reviews and deprescribing have been proposed to improve health status and reduce medicationrelated adverse events and repeat hospitalisations.

- Considerable literature indicates that deprescribing during hospitalisation can be achieved, but little published evidence exists about the duration of deprescribed medications following hospital discharge and the long-term benefits until death.

- This review aimed to identify hospital-initiated deprescribing interventions targeting older people in the last year of life and investigate their effectiveness and sustainability beyond 6 months post-discharge. Many articles did not specify terminal status and others excluded people in the terminal phase of illness.

- Studies generally demonstrated reductions in numbers of potentially inappropriate medications within short timeframes (2-3 months) and decrease in drug-related hospitalisations at 12 months. Evidence of longer-term impact on reducing adverse events or mortality, or improving quality of life or physical functioning, was lacking. Small sample sizes, heterogeneity of interventions and outcomes measures, and design flaws precluded definitive evidence of effectiveness. .

- Further randomised clinical trials are warranted that have larger samples, longer follow-up periods, and objective classification of people as being in the last year of life. 


\section{Introduction}

Evidence suggests older people in their last year of life receive non-essential medicines that can cause discomfort, serious side effects and unnecessary drug interactions [1], or are no longer beneficial given the limited life expectancy. [2,3]. Enabling a "good death" involves allowing people to die without unwarranted medicalisation of the last phase of their life with surgeries, intensive testing, medical procedures and polypharmacy. Continuation of multiple medications where risk of harm outweighs benefit can in fact reduce the quality of their last months of life. But de-prescribing is a complex activity that requires patients and caregivers to understand that discontinuation of chronic medication is not harmful and does not constitute abandonment [4].

The problem of low discontinuation rates of inappropriate medications post-discharge in patients at the end of life [5, 6], and its association with hospital readmission, adverse events and premature death [7] is being increasingly recognised. The detrimental effects of polypharmacy and inappropriate prescribing in hospitalised older patients, and strategies used to mitigate such overuse, have been widely investigated [8]. Poor communication across single-organ specialties and lack of involvement of a single cross-disciplinary pharmacist [9] contributes to the prevalence of polypharmacy and unintended harm in hospitalised patients. Clinical inertia [10] reflecting clinicians' lack of confidence in changing treatment that patients appear to tolerate [11], and desire to avoid family conflict or loss of patient trust may also inhibit uptake of deprescribing.

Strategies for addressing clinical inertia and preventing medication harm, such as appropriate prescribing guidelines, lists of drugs to be avoided [12] and deprescribing protocols based on expert knowledge, have all been widely promulgated $[13,14]$. Recommendations focus on general principles in decreasing the total number of preventive medications for chronic conditions among older people and tailoring the number of symptom-controlling medications [15]. However, some studies deliberately exclude people with limited life expectancy [16-19], and others combine results for people with risk of short-term death in the same category as older people in general, with no distinction between the two groups [20].

Three systematic reviews have investigated deprescribing effectiveness in hospitalised older people (no end of life focus): One review of 31 randomised controlled trials (RCTs), of which half had low risk of bias, found no impact of one-time pharmacist-led medication reviews on 
readmissions, adverse events, quality of life or mortality [21]. Another review of 4 heterogeneous RCTs found that the use of Screening Tool of Older Persons' potentially inappropriate Prescriptions (STOPP) criteria reduced potentially inappropriate medications (PIMs), selected adverse events and health service re-utilisation [22]. A third review found some reduction of PIMs, but reports on clinical outcomes were limited and included studies were generally of low quality and high heterogeneity [23]. A narrative review focused on terminal illness and considered comorbidities, symptom control and drugs to be avoided, but did not include objective measures of effectiveness [24]. To our knowledge, no systematic review has been undertaken to specifically identify effective and sustainable hospital-initiated deprescribing strategies among older people in the last year of life. We sought to fill this gap as the continuing absence of such a review hinders formulation of evidence-based recommendations specific to this highly vulnerable group of patients.

\section{Patients and Methods}

This systematic review investigated interventions associated with sustained adherence to deprescribing among older patients hospitalised near the end of life. For the purpose of this review, 'sustained' was defined as at least six months of follow-up or until death. The methods were agreed prior to the conduct of the review and, due to backlogs in PROSPERO during 2019, the protocol was registered with Open Science Framework (https://osf.io/9mv8d/), before the end of title-abstract and full text screening. Our review follows the AMSTAR 2 guidelines [25], and the TIDieR checklist [26] for intervention description; we chose risk of bias questions to cater for both randomised trials and other interventional studies from several sources (see Critical Appraisal section below) and followed The Cochrane Handbook [27] for risk of bias illustration.

\subsection{Search Strategy}

One author (JC) searched Ovid MEDLINE, the Cochrane Central Register of Controlled Trials (CENTRAL) and Embase. The search string was initially designed in Ovid MEDLINE then translated using the Polyglot Search Translator to be run in the other databases.[28] The search strings were comprised of appropriate subject $(\mathrm{MeSH})$ terms and words, such as deprescribing, OR inappropriate prescribing, OR cease, OR discontinue, OR withdraw AND frail elderly, OR hospitalised OR last, OR limiting AND effectiveness, reduction, 
implementation (Details in Supplement 1, Table S1.2). The searches of the bibliographic databases were supplemented by searching the reference lists of included studies and other relevant review articles. Pairs of reviewers (MC, PS, PF, JA,) independently screened all titles, abstracts and full texts and discrepancies were resolved by discussion or inclusion of a third author. The PROSPERO database and clinical trials registries were also searched to identify further unpublished studies. For study protocols without data, the trials registries and grey literature were searched to identify any published results and one author (PS) contacted at least one of the nominated authors on the protocol. Hand searching of reference lists from potentially eligible articles in systematic reviews followed (MC, PF).

Exclusion criteria were any studies without an intervention or a control group, intervention not initiated in hospital, or not reporting nominated primary outcomes post-discharge. All exclusions were justified and documented by two reviewers (MC, PF) (Supplement 1, Table S1.3). Likewise, reviewer dyads independently examined full texts and agreed on the final selection of eligible studies (PS, MC, PF). We had no restrictions with regards to year of publication or language, but the few foreign language studies found were ineligible based on selection criteria. The initial automated search was conducted in September 2018 and last updated in May 2020 (JC) following the same process.

\subsection{Study populations}

Study populations were patients in the last year of life, as defined by reference of authors to 'terminal', 'palliative', 'near death', 'end of life', 'limited life expectancy' or similar terms or phrases. If these were not present in the abstract or full text, articles were deemed potentially eligible and at the full text screening stage we checked their results tables against the validated Criteria for Screening and Triaging to Appropriate aLternative care (CriSTAL) risk prediction tool. In this way we applied an operational definition of end of life based on the combination of old age and CriSTAL risk factors [29, 30] which reviewers could ascertain from the published study. As examples, if mean or median age was 80 or more years and two CriSTAL risk factors were reported, or age was 65 or more years and four risk factors were reported, then the patients in the study were considered to be near the end of life, and the article was included in the analysis (Supplement 1, Table S1.1).

\subsection{Interventions and comparators}


Interventions were eligible if intended to reduce the number or dose of inappropriate medications to any extent: withdrawal of medicines that are no longer effective/indicated; or substitution of drugs that are inappropriate; or discontinuation of medicines that are not aligned with goals of care; or decreasing total number of potentially inappropriate medications; or dosage reduction/correction or duration adjustment of particular medications to prevent adverse events [31].

The control groups could involve either a different intervention, or usual care (as defined by authors), or modified usual care, or no intervention at all. We did not exclude studies based on follow-up time as our secondary outcomes of interest included any reported outcome timeframe.

\subsection{Study selection}

As the research question was effectiveness, only quantitative studies with control groups were considered for inclusion: randomised controlled trials (RCTs); quasi-randomised trials with a control group; cohorts with a control group; and case-control studies. Systematic reviews were not included but relevant individual RCTs were hand searched from their reference list to assess whether they met inclusion criteria. Excluded studies were those with a pre-post evaluation or qualitative design, lacking a control group, conducted in non-hospital settings (such as residential aged care, or general practice), where the end-of-life status was not declared or inferable by our operational definition, and no reporting of at least one of the outcomes of interest.

\subsection{Data extraction and synthesis}

Study characteristics such as country, year of publication, subject demographics, intervention type and duration, follow-up period, and reported outcomes were extracted by one reviewer (MC) and checked by another (OB) and presented in tables, and another author (PS) confirmed eligibility of the outcomes, all of which attracted $100 \%$ agreement. Intervention descriptions including clinical staff involved were directly extracted from the text. As heterogeneity of outcomes was anticipated, our protocol stated we only intended to conduct meta-analysis where comparability of interventions and outcome measures was possible, otherwise a narrative synthesis would be presented. Proportions or odds ratios with $95 \%$ confidence intervals were to be extracted independently by two reviewers (MC, OB) along 
with reported timeframes, and any inconsistencies resolved through discussion.

\subsection{Critical appraisal}

Two reviewers (PS, OB) independently conducted risk of bias assessments and resolved any discrepancies by discussion, with any outstanding discrepancies resolved by a third reviewer (MC). Criteria used in these assessments were derived from several published tools: Cochrane RoB2 [32] for RCTs and pragmatic interventions, and Cochrane tool [33] and MINORS [34] for cohort or other interventional studies [35] (See protocol at https://osf.io/9mv8d/). Risk of bias assessment was conducted using a web based review platform, SyRF (Systematic Review Facility, https://app.syrf.org.uk/home). For all questions two reviewers independently rated the risk of bias as high, low, or moderate with some concerns (Supplement 2, Table S2.1).

\subsection{Primary and secondary outcomes}

As heterogeneity of outcome measures was anticipated, we agreed a priori to report as primary outcome any single or multiple quantitative indicators of deprescribing effectiveness as reported by included studies: e.g. Mean number or proportion of potentially inappropriate medications (as defined by authors according to: interactions, duplicate classes, not approved or valid indication, no effect on symptom control) that remained discontinued post-discharge; or proportions of patients maintaining discontinuation or dose reduction of at least one medication for at least 6 months.

Secondary outcomes eligible for reporting were: deprescribing measures, as defined above, at shorter follow-up durations (e.g. post-discharge discontinuation rates at $<6$ months); Medication Appropriateness Index (scores) in the first year post-discharge; hospital admissions or visits to the emergency department (ED) any time after discharge; and number or rates of reported medication-related or all-cause adverse events any time after the intervention (details in OSF protocol).

\section{Results}

Seven studies including 1,747 hospital participants in six countries (3 in Europe, 2 in North America, and 2 in Asia/Oceania) met the criteria for inclusion. Only two declared their 
participants' status as terminal [36, 37], while the others qualified as end-of-life after assessing participant characteristics using the CriSTAL tool. Meta-analysis was not performed due to high level of clinical and methodological heterogeneity in intervention modality, outcome measures, and timing of follow-up for outcome ascertainment ranging from the time of discharge to 1 year follow up. There was also substantial heterogeneity in the intervention components, participating staff profiles, and duration of interventions. Hence, we decided to report findings in a narrative form. Most exclusions after full text review were due to ineligible study design, absence of an end-of-life focus, or non-hospital setting. Further details of reasons for exclusion are shown in Supplement 1, Table S1.3 and PRISMA diagram in Figure 1.

$<$ Figure 1 about here $>$ PRISMA diagram for study screening and selection, and reasons for exclusion.

\subsection{Study characteristics}

Five studies were RCTs [38-42], one was a retrospective non-randomised controlled cohort study [43] and another was a prospective non-randomised controlled trial [36]. The mean age of participants in six studies ranged from 82.7 to 86.6 years and with larger representation of women (59\%-71\%), with the one exception of a retrospective cohort which enrolled younger participants (mean 71 years) and mostly (55\%) men (Table 1). All studies reported wardbased interventions and two also included further interventions in outpatients clinics [39] or in the community [40]. Maintenance of drug cessation for at least 6 months was the primary outcome in only one study [38], and maintenance of PIMs at 2-3 months post-discharge was the secondary outcome in two $[39,41]$. Health service utilisation after discharge and adverse events, including mortality, were the next most commonly reported outcomes.

\section{$<$ TABLE 1 ABOUT HERE $>$ Table 1. Relevant hospital studies, methods and intervention characteristics 1990 to 2020}

\subsection{Study quality}

The risk of bias of RCT designs and conduct was moderate to low across most domains. Overall, sample sizes were generally low (range 110 to 436, median 368, IQR 161-395), however, two studies recruited over 400 participants [40, 41]. Two studies did not adequately 
conceal allocation [40, 41], one made no comment on allocation [43], and the appropriateness of concealment was uncertain in another [39]. Only the two non-randomised studies clearly reported following an intention to treat analysis [36, 43] whereas all RCTs either failed to conduct or report it, and none involved blinded outcome assessors. Despite this, baseline characteristics were predominantly balanced between groups. Two studies [36, 41] with available protocols did not report their pre-specified outcome measures and two [40, 41] also reported on outcomes that were not pre-specified.

Most studies had low patient attrition, between $0 \%$ and $8 \%$, although one study [39] reported loss to follow-up of $20 \%$, mostly due to deceased participants. Most studies did not report a conflict of interest and sources of funding were predominantly academic or government, and no study was funded by a pharmaceutical company. A risk of bias summary and graph are presented in Supplement 2, Figure 2.1.

\subsection{Intervention characteristics}

Applying the TIDieR checklist [26], the intervention descriptions overall lacked detail in terms of duration, frequency, staff involvement and characteristics of the care provided to the control groups, which made it difficult to compare effectiveness of different interventions (Table 1 and Supplement 2, Table S2.2). In five studies the intervention was described as usual care enhanced with other activities or personnel [38-40, 42, 43]. The most common feature of interventions that went beyond usual care, as reported in five studies [39-43], was the participation of a pharmacist in the medication review during hospitalisation before patient discharge. The next most frequently involved team member in intervention groups was the ward physician or internist $[39,40,42,43]$. In addition to the core staff, two interventions engaged the geriatrician $[38,41]$, one intervention integrated the community pharmacist coordinator from hospital into an aged care facility, [39], another involved a nurse in identifying candidates suitable for deprescribing intervention [38] and one comprised a multidisciplinary team with allied health workers and nurses participating in deprescribing discussions [41]. Only two interventions included an education component that engaged patients[40, 43].

Where described, target medications were predominantly multiple drug classes used to manage chronic irreversible illness, such as antiarrhythmics, neuroleptics, antidepressants, psychotropics (sedatives, anticholinergic, opioids), proton pump inhibitors, anticonvulsants 
and hypoglycaemic agents. Only one study targeted a single medication class: statins [36], and one did not report target medications but instead appeared to target overall prescribing [39].

Interventions comprising medication review were implemented as either a one-off event, or the duration was not reported. One study conducted a second medication review at discharge [44], and one repeated the intervention at two months by telephone [40]. Follow-up periods varied from less than 3 months [39, 41] to six-month [43] and 12-months [36, 38, 40] (Table 1). While all interventions were commenced during the hospitalisation, three studies included patients who returned for follow-up at outpatient clinics [36, 39, 40].

The control group was often poorly described as usual care, with an indication that either a single professional, such as a geriatrician, or ward staff conducted less comprehensive assessments and medication reviews. Two of the studies did not offer a description of the staff delivering the usual care, $[36,41]$ and the cohort study defined control patients as those who were not contactable or who refused to participate [43].

\subsection{Outcomes at $\geq 6$ months}

Only two studies followed participants up to 12 months $[38,40]$, and two others followed for 7-8 months $[36,43]$. As shown in Table 2, sustainable discontinuation (beyond six months) was only reported by one RCT where a geriatrician-led medication review using STOPP criteria achieved (62\%) cessation rates of PIMs at one year after intervention [38]; but this reduction was not significantly different from the control group (57\%) who were managed by the attending physician and hospital pharmacist [38].

Only one RCT reported 12-month health service use and found a significantly lower proportion of medication-related readmissions in the intervention group versus the control group (4.9\% vs. $24 \%$, OR 0.20 (95\% CI $0.10-0.41$; p $<0.05)$ and markedly lower proportions visiting the ED post discharge $(26.9 \%$ vs. $50 \%$, OR 0.53 , 95\%CI $0.37-0.75$; $<<0.05)$. [40] However, the overall readmission rate at 12 months was no different between the two groups (58.2\% vs. 59.1\%, p>0.05). This was a multi-faceted intervention with comprehensive medication review, patient education and pre-discharge counselling by a pharmacist, discharge to GP care and 2-month telephone follow-up. The non-RCT with 8-month followup also found no significant differences in unplanned hospital admissions $(21.9 \%$ and $18.9 \%$ 
in intervention and control groups respectively) [43].

\section{$<$ TABLE 2 ABOUT HERE $>$ Table 2. Estimates of sustainable effectiveness and other impact of hospital-initiated deprescribing by duration of follow-up}

Two studies with follow-up $>6$ months reported mortality as an outcome: a multicomponent intervention with 12-month follow-up [40], and a multi-component intervention for patients with hip fracture with mean follow-up of eight months [43]. Neither found a significant difference between groups at the end of follow-up, and the latter trial also showed no difference in the proportion of patients experiencing new fractures [43].

\subsection{Outcomes under 6 months}

The total number of medications was significantly reduced at 3-months follow-up in a RCT in which the existing team of ward physician, hospital pharmacist, and nurses, coupled with a research physician, applied STOPPFrail recommendations [42]. Compared with usual care, statistically significant lower proportions of patients on $\geq 1$ PIM and of those lacking indications for one or more medication was achieved at three months following a multidisciplinary team assessment, team conference and pharmacist review within 72 hours [41]. Significant reductions in PIMs were also observed at two months in an intervention group that engaged a pharmacist transition coordinator, held a case conference with physician and pharmacists before discharge, and involved a community pharmacist on discharge to the nursing home [39]. However, only $20 \%$ of the original sample was assessed at follow-up. In three studies, there was no difference between intervention and control groups in the proportions of patients experiencing adverse events or falls, or new cardiovascular events at either two or three months $[36,39,42]$. Our secondary outcome of quality of life was reported in only one non-randomised study [37-41, 43], wherein discontinuation of statins exerted a favourable effect on quality of life (OR 0.26 , 95\%CI 0.02-0.50). However, this non randomised trial failed to adequately describe its intervention [36] so no reliable conclusions can be drawn.

\section{Conclusions}

This review suggests that multi-component, multidisciplinary deprescribing interventions for older people nearing the end of life may be effective at reducing potentially inappropriate 
prescribing or the number of prescribed medications in the short-term. However, evidence is lacking as to whether they are associated with either a sustained drug cessation at six or twelve months post-discharge or a decrease adverse events or hospital admissions beyond the immediate post-discharge period. Small sample sizes, short follow-up periods, and heterogeneity of interventions and outcomes of included studies do not allow definitive statements to be made on whether deprescribing strategies were effective or sustained in our target group.

Multi-faceted interventions (such as medication withdrawal plan using standard tools, communication to attending physician, and nurse monitors [37], or medication reviews and case conference with the hospital management team [41] supplemented with sharing of information with residential aged care nurses and community pharmacists [39]); and involvement of interdisciplinary groups within the hospital (hospital pharmacist, ward physician, ward nurses, and/or social worker, physiotherapist, dietician) and outside the hospital (community pharmacist, transition coordinator, research physician), tended to achieve better results than once off activities or strategies with single professionals or smaller teams. However, the limited descriptions in published articles of usual care and of the precise tasks and activities undertaken within intervention groups prevented identification of individual components that may be the strongest and most important contributors to deprescribing effectiveness.

\section{Expert opinion}

\subsection{Lack of focus on end of life care}

Our review adds to the evidence base by focussing on patients nearing the end of life who, as shown by the small number of included studies, are markedly under-represented in deprescribing trials. While it is generally accepted that polypharmacy should be avoided [45] and prescribing should be reconciled with management goals for patients with comorbidities and limited life expectancy $[24,46]$, our results suggest that few deprescribing interventions focus on people in the last year of life. Only two studies explicitly identified or targeted those at end-of life [36, 42], while the remaining studies were identified using the CriSTAL tool. We were able to find four studies with a 6-month or greater follow-up, two of which had 12month follow up, but only one study assessed medication use 6 months after deprescribing intervention. The practicalities of enrolling or following up terminal patients were not 
described in the included studies.

\subsection{The quality of evidence for sustainability}

In terms of health service utilisation, a multi-component intervention significantly reduced 12-month drug-related readmissions and ED re-attendances. However, there was no evidence of reduction in unplanned all-cause hospital admissions or of mortality at any time point. regardless of sample size and type of intervention. Studies that reported significant reductions in total numbers of prescriptions and significantly lower proportions of patients on inappropriate medications up to 3 months involved interventions that required additional clinical team members which may not be feasible in routine practice.

Overall included studies were assessed as having moderate risk of bias due to lack of random assignment or flaws in allocation concealment, but short follow-up periods limited evaluation of sustainability of the primary outcome. The heterogeneity of intervention approaches, duration of follow-up and outcome measurement precluded meta-analysis of pooled data.

The complex and time-consuming nature of deprescribing is challenging [47] and may contribute to the small number of well conducted studies of hospital-based interventions. Some propose that implicit clinical judgment in deciding prescribing appropriateness is more feasible than applying cumbersome deprescribing tools that require explicit criteria [48]. Others suggest risk stratification algorithms that guide the targeting of deprescribing to high risk individuals or high risk drug classes may yield better clinical outcomes [49]. In our review, three studies used well-cited objective criteria checklists (STOPP [38, 42], MAI [39]) to decide on inappropriateness and the need to deprescribe. Unfortunately, the generic descriptions of interventions in the other studies (e.g. comprehensive/optimal medication review) $[36,40,41,43]$ and absent $[36,41]$ or minimalist descriptions of usual care in the control groups of three studies $[39,42,43]$ make it difficult to deduce the extent to which these findings can be replicated in routine practice.

The withdrawal of medicines with no indications or no alignment with goals of care is an imperative [31], and practical guidance for deprescribing in older patients in routine practice is required [50]. However, whether deprescribing is sustained at or beyond six months for patients near the end of life is yet to be demonstrated in well-designed trials. 


\subsection{Comparison with other studies}

Lack of evidence for long-term effectiveness of inpatient medication review was also evident in a recent systematic review reporting reductions in emergency re-attendances from 30 days to one year, but no evidence of decreased mortality or hospital readmission [51].The absence of longer term follow-up precludes comments around sustainability.

Four recent reviews that examined older patients in general deserve comment. One reported deprescribing interventions in older people across settings, predominantly nursing homes, and reported no significant impact on either quality of life or satisfaction with care,[52] although many of the included articles had high risk of bias. Another review of deprescribing preventive medicines in advanced illness, which included uncontrolled studies and retrospective analyses without interventions, reported that deprescribing statins could improve quality of life but deprescribing warfarin could be harmful [53]. A third review, which defined life-limiting illness on the basis of single conditions and included nursing home residents, suggested that deprescribing interventions could reduce medication inappropriateness at discharge and up to 24 months later [54]. However, their conclusions were derived from multiple pre-post studies, not RCTs. Computerised medication review systems have been helpful in preventing inappropriate prescribing of older inpatients but studies have not included patients at the end-of-life, thus limiting their generalisability to this group [55].

While palliative care provided to terminally ill patients dying in hospital systematises routine discussions of deprescribing with patients, such discussions have not been incorporated or described in the intervention components of studies reviewed here, perhaps with the exception of the two that conducted self-efficacy education of patients and/or families on reasons for medication changes and treatment goals [40].

In summary, to the best of our knowledge, there is insufficient evidence to show if any deprescribing intervention in older hospitalised patients near the end of life is effective and sustained over the longer-term following hospital discharge.

\subsection{Limitations}

Our review is limited by the heterogeneity of intervention designs and outcome measures 
which precluded meta-analysis or attribution of effectiveness to individual intervention components. As we adopted the last year of life as a pragmatic definition [56] for study selection, we risked excluding some relevant publications that did not characterise their subjects as being in this phase of their life. To bridge that gap we used an additional operational definition of near end-of-life, aligned with validated clinical CriSTAL criteria rather than based on timeframe or care goals, to uncover interventions that targeted or included terminally ill older patients. In two studies [40, 41] the reference to multiple risk factors suggested the majority of the study population were terminal, although the distribution of individual risk factors could not be quantified to definitely confirm each participant met our inclusion criteria. Due to resource constraints we did not search the CINAHL database and this may potentially have led to missing some eligible studies.

\subsection{Implications for practice}

While the findings of this review suggest improvements in identifying and reducing PIMs in older patients near the end of life, whether deprescribing interventions initiated in hospital achieve long-term benefits in measurable patient outcomes remains uncertain, and hence more evidence is required before they can be recommended for use in routine practice

\subsection{Implications for future research}

The importance of deprescribing non-essential medications in older people receiving end of life care [36] is interlinked with the need for advance care planning. Expert consensus guidelines have identified polypharmacy late in life as a priority for education and research [47], with benzodiazepines, atypical antipsychotics, statins, tricyclic antidepressants, and proton pump inhibitors comprising priority medication groups for deprescribing [57]. Despite this, our findings suggest that limited research has specifically focused on deprescribing in older people near the end of life, arguably a group who might benefit greatly from proactive deprescribing. Only two out of seven eligible studies in this review explicitly reported that recruited patients had advanced life-limiting illness [36, 42], hence our need to use a proxy operational definition to confirm eligibility. Objective tools such as CriSTAL are available to assist clinicians in identifying patients nearing end of life who can then be targeted for deprescribing interventions aimed at preventing continuation of drugs that are no longer indicated or aligned with treatment goals in the last months of life) [14]. 
Significant reductions in post-discharge hospitalisations and emergency visits as a result of hospital-initiated deprescribing interventions, were found in two of the included RCTs [39, 40] - findings which warrant further investigation in more high quality trials. Despite insufficient information on medication-related activities undertaken within the control groups, the finding of effectiveness of multicomponent deprescribing interventions delivered by multidisciplinary teams is promising and deserves further investigation in more rigorous study designs. Since nurses spend considerably more time with patients during hospitalisation than other types of clinicians, and were involved in the those interventions showing promise [39, 41], future studies might consider investigating an expanded scope of practice for nurses in optimising use of medications. Medication overtreatment and gaps in the drug safety profile of older people dying of natural causes remain unaddressed problems. Other aspects of deprescribing such as the impact on quality of life, the influence of family involvement in the decision to deprescribe [58] and the benefits of more targeted patient education [59] also deserve further study. We recommend that future trials of deprescribing near end of life should include clear definitions of end of life, randomisation, intention to treat analysis, complete descriptions of interventions and usual care, and sufficient follow-up (for at least six months or preferably until death) in assessing sustainability of deprescribing outcomes over the longer term.

\section{Acknowledgments}

We thank Associate Professor Mark Jones for his advice on data extraction in the early stages of the project, Dr Alex Bannach-Brown for setting up the risk of bias platform, and the Bond University librarians for their assistance with interlibrary loan acquisitions.

\section{Author contributions}

Design and protocol: MC, PS, JC, IS. Searches: JC, MC. Screening: PF, MC, JA, PS, OB, SS. Data extraction: MC, OB, PF. Risk of Bias: PS, OB. Analysis: MC, OB. Interpretation: MC, IS, OB, PS. Manuscript writing: MC, IS, OB. Revisions and approval of last version: All authors.

\section{Funding}

No funding other than our salaries and in-kind support from medical and pharmacy trainees was available to conduct this review. Salaries of staff involved in this review are paid from 
the academic or clinical institutions to which they are affiliated.

\section{Declaration of interests}

M Cardona previously designed and validated the CriSTAL tool used to identify people at risk of short-term death. She derives no financial or other benefits from the use of the tool in any setting. The authors have no other relevant affiliations or financial involvement with any organization or entity with a financial interest in or financial conflict with the subject matter or materials discussed in the manuscript. This includes employment, consultancies, honoraria, stock ownership or options, expert testimony, grants or patents received or pending, or royalties.

\section{Reviewer disclosures}

Peer reviewers on this manuscript have no relevant financial or other relationships to disclose. 


\section{References}

1. Maher RL, Hanlon J, Hajjar ER. Clinical consequences of polypharmacy in elderly. Expert Opin Drug Saf. 2014;13(1):57-65.

2. Holmes HM, Todd A. Evidence-based deprescribing of statins in patients with advanced illness. JAMA internal medicine. 2015 May;175(5):701-2.

3. Maddison AR, Fisher J, Johnston G. Preventive medication use among persons with limited life expectancy. Progress in palliative care. $2011 \mathrm{Jan} ; 19(1): 15-21$.

4. Reeve E, Low LF, Hilmer SN. Beliefs and attitudes of older adults and carers about deprescribing of medications: a qualitative focus group study. The British journal of general practice : the journal of the Royal College of General Practitioners. 2016 Aug;66(649): e55260 .

5. Paque K, De Schreye R, Elseviers M, Vander Stichele R, Pardon K, Dilles T, et al. Discontinuation of medications at the end of life: A population study in Belgium, based on linked administrative databases. Br J Clin Pharmacol. 2019 04;85(4):827-37.

6. Pasina L, Brignolo Ottolini B, Cortesi L, Tettamanti M, Franchi C, Marengoni A, et al. Need for Deprescribing in Hospital Elderly Patients Discharged with a Limited Life Expectancy: The REPOSI Study. Med Princ Pract. 2019;28(6):501-8.

7. Weir DL, Lee TC, McDonald EG, Motulsky A, Abrahamowicz M, Morgan S, et al. Both New and Chronic Potentially Inappropriate Medications Continued at Hospital Discharge Are Associated With Increased Risk of Adverse Events. Journal of the American Geriatrics Society. 2020 Mar 31;31:31.

8. Davies LE, Spiers G, Kingston A, Todd A, Adamson J, Hanratty B. Adverse Outcomes of Polypharmacy in Older People: Systematic Review of Reviews. Journal of the American Medical Directors Association. 2020 Feb;21(2):181-7.

9. Sherman JJ, Davis L, Daniels K. Addressing the Polypharmacy Conundrum. US Pharmacist. 2017;42(6):HS-14-HS-20.

10. Steinman MA, Landefeld CS. Overcoming Inertia to Improve Medication Use and Deprescribing. Jama. 2018 Nov 13;320(18):1867-9.

11. Djatche L, Lee S, Singer D, Hegarty SE, Lombardi M, Maio V. How confident are physicians in deprescribing for the elderly and what barriers prevent deprescribing? J Clin Pharm Ther. 2018 Aug;43(4):550-5.

12. O'Mahony D, O'Connor MN. Pharmacotherapy at the end-of-life. Age Ageing. 2011 Jul;40(4):419-22.

13. Farrell B, Mangin D. Deprescribing Is an Essential Part of Good Prescribing. American family physician. 2019 Jan 1;99(1):7-9.

14. Scott IA, Anderson K, Freeman C. Evidence-based deprescribing: Reversing the tide of potentially inappropriate polypharmacy. Journal of clinical outcomes management. 2016 2016;23(8):359 69.

15. Currow DC, Stevenson JP, Abernethy AP, Plummer J, Shelby-James TM. Prescribing in palliative care as death approaches. J Am Geriatr Soc. 2007 Apr;55(4):590-5.

16. Low LL, Tan SY, Ng MJ, Tay WY, Ng LB, Balasubramaniam K, et al. Applying the Integrated Practice Unit Concept to a Modified Virtual Ward Model of Care for Patients at Highest Risk of Readmission: A Randomized Controlled Trial. PLoS ONE. 2017;12(1):e0168757.

17. Franchi C, Tettamanti M, Djade CD, Pasina L, Mannucci PM, Onder G, et al. Elearning in order to improve drug prescription for hospitalized older patients: a clusterrandomized controlled study. Br J Clin Pharmacol. 2016 Jul;82(1):53-63.

18. Lim WS, Low HN, Chan SP, Chen HN, Ding YY, Tan TL. Impact of a pharmacist consult clinic on a hospital-based geriatric outpatient clinic in Singapore. Annals of the Academy of Medicine, Singapore. 2004 Mar;33(2):220-7. 
19. Schmader KE, Hanlon JT, Pieper CF, Sloane R, Ruby CM, Twersky J, et al. Effects of geriatric evaluation and management on adverse drug reactions and suboptimal prescribing in the frail elderly. The American journal of medicine. 2004 Mar 15;116(6):394-401.

20. Malik NA, Panagoda WK, Tan FYH, Pang WY. Frailty-guided management model for severely frail older adults in an acute hospital setting. European geriatric medicine. 2017;8:S103.

21. Huiskes VJ, Burger DM, van den Ende CH, van den Bemt BJ. Effectiveness of medication review: a systematic review and meta-analysis of randomized controlled trials. BMC Fam Pract. 2017 Jan 17;18(1):5.

22. Hill-Taylor B, Walsh KA, Stewart S, Hayden J, Byrne S, Sketris IS. Effectiveness of the STOPP/START (Screening Tool of Older Persons' potentially inappropriate Prescriptions/Screening Tool to Alert doctors to the Right Treatment) criteria: systematic review and meta-analysis of randomized controlled studies. J Clin Pharm Ther. 2016 Apr;41(2):158-69.

23. Thillainadesan J, Gnjidic D, Green S, Hilmer SN. Impact of Deprescribing Interventions in Older Hospitalised Patients on Prescribing and Clinical Outcomes: A Systematic Review of Randomised Trials. Drugs Aging. 2018 Apr;35(4):303-19.

24. Cruz-Jentoft AJ, Boland B. Drug therapy optimization at the end of life. Drugs \& Aging. 2012;29(6):511-21.

25. Shea BJ, Reeves BC, Wells G, Thuku M, Hamel C, Moran J, et al. AMSTAR 2: a critical appraisal tool for systematic reviews that include randomised or non-randomised studies of healthcare interventions, or both. BMJ. 2017;358:j4008.

26. Hoffmann TC, Glasziou PP, Boutron I, Milne R, Perera R, Moher D, et al. Better reporting of interventions: template for intervention description and replication (TIDieR) checklist and guide. Bmj. 2014 Mar 7;348:g1687.

27. Cochrane Statistical Methods Group and the Cochrane Bias Methods Group. Chapter 8: Assessing risk of bias in included studies. In: Julian PT Higgins, Douglas G Altman, Jonathan AC Sterne, editors.

28. Clark JM, Sanders S, Carter M, Honeyman D, Cleo G, Auld Y, et al. Improving the translation of search strategies using the Polyglot Search Translator: a randomized controlled trial. Journal of the Medical Library Association : JMLA. 2020 Apr;108(2):195-207.

29. Cardona-Morrell M, Hillman K. Development of a tool for defining and identifying the dying patient in hospital: Criteria for Screening and Triaging to Appropriate aLternative care (CriSTAL). BMJ supportive \& palliative care. 2015 Mar;5(1):78-90.

30. Cardona M, Lewis ET, Kristensen MR, Skjot-Arkil H, Ekmann AA, Nygaard HH, et al. Predictive validity of the CriSTAL tool for short-term mortality in older people presenting at Emergency Departments: a prospective study. European geriatric medicine. 2018;9(6):891901.

31. Frank C, Weir E. Deprescribing for older patients. CMAJ. 2014;186(18):1369-76.

32. Sterne JAC, Savovic J, Page MJ, Elbers RG, Blencowe NS, Boutron I, et al. RoB 2: a revised tool for assessing risk of bias in randomised trials. Bmj. 2019 Aug 28;366:14898. 33. Jonathan AC Sterne, Miguel A Hernán, Alexandra McAleenan, Barnaby C Reeves, Julian PT Higgins. Chapter 25: Assessing risk of bias in a non-randomized study. Cochrane Training; 2019.

34. Slim K, Nini E, Forestier D, Kwiatkowski F, Panis Y, Chipponi J. Methodological index for non-randomized studies (minors): development and validation of a new instrument. ANZ journal of surgery. 2003 Sep;73(9):712-6.

35. Page MJ, McKenzie JE, Higgins JPT. Tools for assessing risk of reporting biases in studies and syntheses of studies: a systematic review. BMJ Open. 2018;8(3):e019703.

36. Kutner JS, Blatchford PJ, Taylor DH, Jr., Ritchie CS, Bull JH, Fairclough DL, et al. Safety and benefit of discontinuing statin therapy in the setting of advanced, life-limiting illness: a randomized clinical trial. JAMA internal medicine. 2015 May;175(5):691-700. 
37. Curtin D, Jennings E, Daunt R, Curtin S, Randles M, Gallagher P, et al. Deprescribing in Older People Approaching End of Life: A Randomized Controlled Trial Using STOPPFrail Criteria. Journal of the American Geriatrics Society. 2020 2020/04/01;68(4):762-9.

38. Dalleur O, Boland B, Losseau C, Henrard S, Wouters D, Speybroeck N, et al. Reduction of potentially inappropriate medications using the STOPP criteria in frail older inpatients: a randomised controlled study. Drugs Aging. 2014 Apr;31(4):291-8.

39. Crotty M, Rowett D, Spurling L, Giles LC, Phillips PA. Does the addition of a pharmacist transition coordinator improve evidence-based medication management and health outcomes in older adults moving from the hospital to a long-term care facility? Results of a randomized, controlled trial. Am J Geriatr Pharmacother. 2004 Dec;2(4):257-64.

40. Gillespie U, Alassaad A, Henrohn D, Garmo H, Hammarlund-Udenaes M, Toss H, et al. A comprehensive pharmacist intervention to reduce morbidity in patients 80 years or older: a randomized controlled trial. Archives of internal medicine. 2009 May 11;169(9):894-900.

41. Owens NJ, Sherburne NJ, Silliman RA, Fretwell MD. The Senior Care Study. The optimal use of medications in acutely ill older patients. J Am Geriatr Soc. 1990 Oct;38(10):1082-7.

42. Curtin D, Jennings E, Daunt R, Curtin S, Randles M, Gallagher P, et al. Deprescribing in Older People Approaching End of Life: A Randomized Controlled Trial Using STOPPFrail Criteria. J Am Geriatr Soc. 2020 Apr;68(4):762-9.

43. Komagamine J, Hagane K. Intervention to improve the appropriate use of polypharmacy for older patients with hip fractures: an observational study. BMC Geriatr. 2017 Dec 16;17(1):288.

44. Wehling M, Burkhardt H, Kuhn-Thiel A, Pazan F, Throm C, Weiss C, et al. VALFORTA: a randomised trial to validate the FORTA (Fit fOR The Aged) classification. Age Ageing. 2016 Mar;45(2):262-7.

45. Mangin D, Bahat G, Golomb BA, Mallery LH, Moorhouse P, Onder G, et al. International Group for Reducing Inappropriate Medication Use \& Polypharmacy (IGRIMUP): Position Statement and 10 Recommendations for Action. Drugs \& aging. 2018 2018/07//;35(7):575-87.

46. Gardner E. Deprescribing in end-of-life care. Journal of Prescribing Practice. 2019;1(11).

47. Muscedere J, Kim P, Aitken P, Gaucher M, Osborn R, Farrell B, et al. Proceedings of the Canadian Frailty Network Summit: Medication Optimization for Frail Older Canadians, Toronto, Monday April 24, 2017. Can Geriatr J. 2017;20(4):253-63.

48. Cheong TTS, Alhamid SM, Li FY, Ang STW, Lim KHJ. Improving prescribing for older patients - 'Yes S-I-R-E!'. Singapore Med J. 2019 Jun;60(6):298-302.

49. Donovan M, O'Connell M, Byrne S. Identifying methods to prioritise patients for deprescribing using a systematic review of deprescribing algorithms in elderly patients. International Journal of Clinical Pharmacy. 2019;41(1):291.

50. Linsky A, Meterko M, Stolzmann K, Simon SR. Supporting medication discontinuation: provider preferences for interventions to facilitate deprescribing. BMC Health Serv Res. 2017;17(1):447-.

51. Christensen M, Lundh A. Medication review in hospitalised patients to reduce morbidity and mortality. Cochrane Database of Systematic Reviews. 2016(2).

52. Pruskowski JA, Springer S, Thorpe CT, Klein-Fedyshin M, Handler SM. Does Deprescribing Improve Quality of Life? A Systematic Review of the Literature. Drugs Aging. 2019 Dec;36(12):1097-110.

53. Narayan SW, Nishtala PS. Discontinuation of Preventive Medicines in Older People with Limited Life Expectancy: A Systematic Review. Drugs Aging. 2017 Oct;34(10):767-76. 54. Shrestha S, Poudel A, Steadman K, Nissen L. Outcomes of deprescribing interventions in older patients with life-limiting illness and limited life expectancy: A systematic review. Br J Clin Pharmacol. 2019 2019/09/04;n/a(n/a). 
55. Dalton K, O'Brien G, O'Mahony D, Byrne S. Computerised interventions designed to reduce potentially inappropriate prescribing in hospitalised older adults: a systematic review and meta-analysis. Age Ageing. 2018 Sep 1;47(5):670-8.

56. The Norwegian National Research Ethics Committees. Persons with limited life expectancy. 2015 [cited 2020 January]; Available from:

https://www.etikkom.no/en/library/topics/research-on-particular-groups/persons-with-limitedlife-expectancy/

57. Farrell B, Tsang C, Raman-Wilms L, Irving H, Conklin J, Pottie K. What are priorities for deprescribing for elderly patients? Capturing the voice of practitioners: a modified delphi process. PLoS ONE. 2015;10(4):e0122246.

58. Noble BN, Izumi S, Tjia J, Ku I, Kadoyama K, Furuno JP. Patient and family involvement in medication decisions on discharge to hospice care. Journal of the American Geriatrics Society. 2019;67:S145.

59. Nowbahari E, Bigot A, Maillot F, Antier D, Foucault-Fruchard L. Reassessment of inappropriate prescriptions of proton pump inhibitors in elderly in-patients: It's time to take action. Annales Pharmaceutiques Francaises. 2020;78(2):150-7.

*5. Paque. Large linked population study showing discontinuation of medications at the end of life is feasible but more is needed

**14. Scott. A comprehensive narrative review with recommendations for older comorbid patients

*30 Cardona. Validation study of a tool for objective identification of end-of-life status

**36. Curtin. A very well designed trial of multidisciplinary intervention

*40. Gillespie. A trial with sufficient follow-up measuring multiple relevant outcomes 
Table 1. Relevant hospital studies, methods and intervention characteristics 1990 to $2020, N=7$ studies

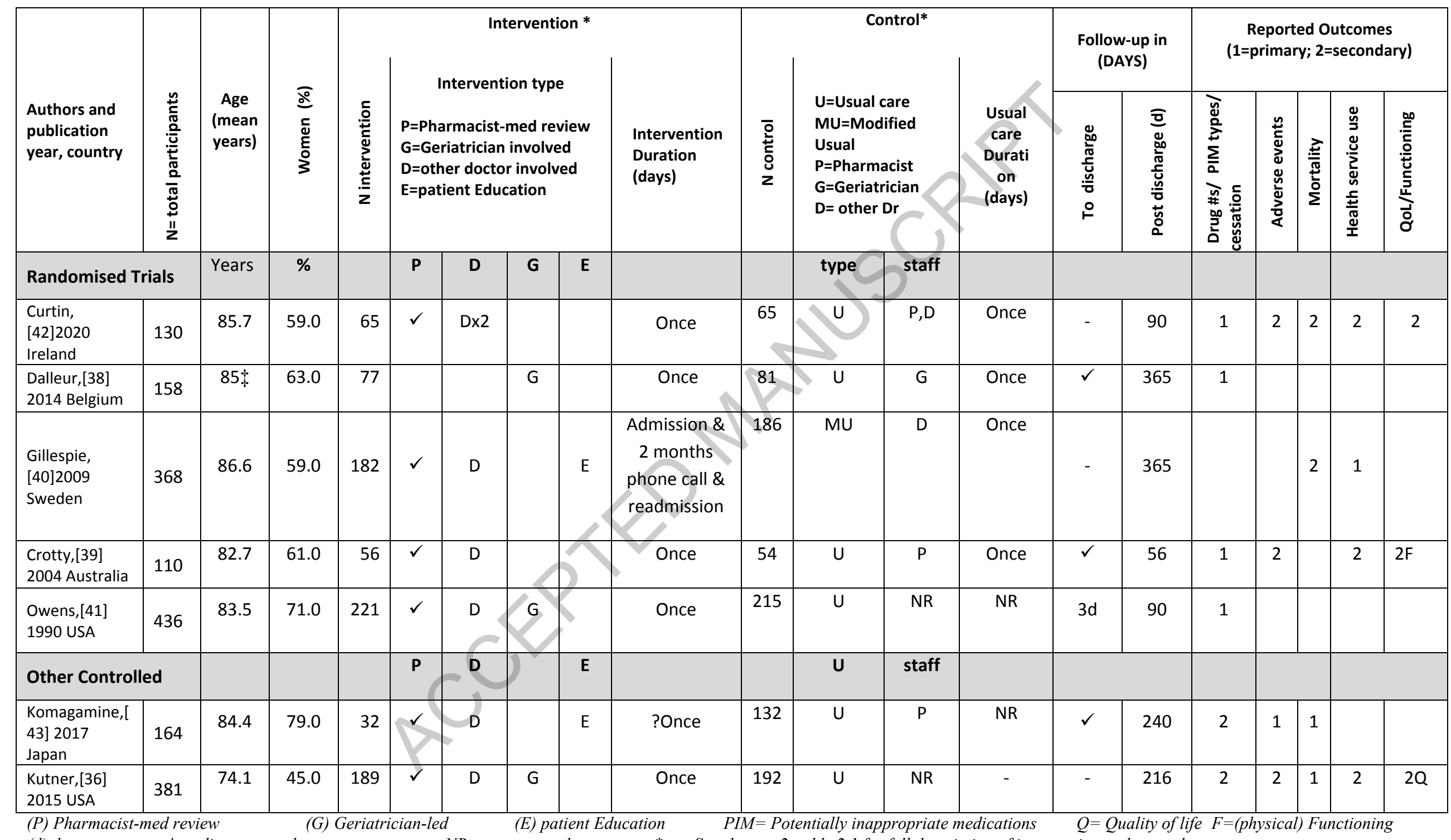

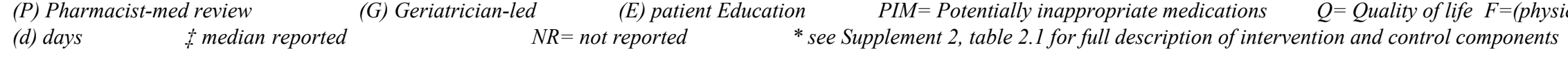


Table 2. Estimates of sustainable effectiveness and other impact of hospital-initiated deprescribing by duration of follow-up ( $N=7$ )

\begin{tabular}{|c|c|c|c|c|c|c|c|c|}
\hline Author, date & 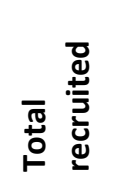 & 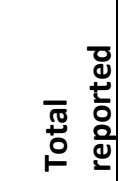 & 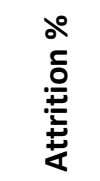 & Indicator description & Timing & $\begin{array}{l}\text { Control } \\
\text { estimate } \%(n / N)\end{array}$ & $\begin{array}{c}\text { Intervention } \\
\text { estimate \% } \\
(n / N)\end{array}$ & $\begin{array}{c}\text { Estimate of } \\
\text { Difference }^{a} \\
\text { and/or } p \text { value }\end{array}$ \\
\hline \multicolumn{9}{|l|}{$\begin{array}{l}\text { Drug cessation or } \\
\text { improvement }\end{array}$} \\
\hline Dalleur,[38] 2014 & 50 & 50 & 0 & Drug cessation PIM\% remained & @ 12 months & $57.0(14 / 24)$ & $62.0(16 / 26)$ & 0.999 \\
\hline Dalleur,[38] 2014 & 50 & 50 & 0 & Restarting rate post-discharge & @ 12 months & $43.0(10 / 24)$ & $38.0(10 / 26)$ & 0.999 \\
\hline Curtin,[42] 2020 & 130 & 130 & 0 & Reduction in number of prescriptions & @3 months & -0.36 (SD 2.60) & $-2.6(S D 2.73)$ & $<0.001$ \\
\hline \multicolumn{9}{|l|}{$\begin{array}{l}\text { Inappropriate } \\
\text { medications }\end{array}$} \\
\hline Owens,[41] 1990 & 436 & 436 & 0 & $\geq 1$ inappropriate medication choice & @ 3 months & $37.0(80 / 215)$ & $20.0(44 / 221)$ & $<0.005$ \\
\hline Crotty,[39] 2004 & 110 & 88 & 20.0 & $\begin{array}{l}\text { Overall \% with inappropriate } \\
\text { medication rating (MAI) Index }\end{array}$ & @2 months & $6.5(3.9-9.3)$ & $2.5(1.4-3.7)$ & 0.007 \\
\hline Owens,[41] 1990 & 436 & 436 & 0 & No indication for multiple medications & @ 3 months & $19.1(41.215)$ & $11.3(25 / 221)$ & 0.025 \\
\hline Crotty,[39] 2004 & 110 & 110 & 0 & No indication for medication & @ 2 months & $14.8(18 / 56)$ & $32.1(8 / 54)$ & 0.043 \\
\hline \multicolumn{9}{|l|}{$\begin{array}{l}\text { Polypharmacy } \\
\text { prevalence }\end{array}$} \\
\hline Owens,[41] 1990 & 436 & 436 & 0 & $\begin{array}{l}\% \text { increased number of medications } \\
\text { from admission to }\end{array}$ & @3 months & $39.0(84 / 215)$ & $40.0(88 / 221)$ & $>0.05$ \\
\hline Owens,[41] 1990 & 436 & 436 & 0 & $\begin{array}{l}\% \text { increased number of medications } \\
\text { from admission to }\end{array}$ & @ 6 weeks & $43.0(92 / 215)$ & $40.0(88 / 221)$ & $>0.05$ \\
\hline \multicolumn{9}{|l|}{ Adverse events } \\
\hline Curtin,[42] 2020 & 130 & 130 & 0 & Adverse events: falls \% & @ 3 months & $21.5(14 / 65)$ & $21.5(14 / 65)$ & 0.75 \\
\hline Crotty,[39] 2004 & 110 & 88 & 20.0 & adverse events: falls \% & @ 2 months & $36.4(16 / 44)$ & $43.2(19 / 44)$ & 0.51 \\
\hline Crotty,[39] 2004 & 110 & 88 & 20.0 & overall adverse drug events \% & @ 2 months & $45.5(20 / 44)$ & $43.2(19 / 44)$ & 0.83 \\
\hline Kutner,[36] 2015 & 381 & 371 & 2.6 & First cardiovascular event \% & @ 7 months & $5.7(11 / 192)$ & $6.9(13 / 189)$ & 0.64 \\
\hline $\begin{array}{l}\text { Komagamine,[43] } \\
2017\end{array}$ & 164 & 158 & 3.7 & Adverse events: any new fracture & @ 8 months & $11.4(15 / 132)$ & $9.4(3 / 32)$ & 1.00 \\
\hline \multicolumn{9}{|l|}{ Health service use } \\
\hline Gillespie,[40] 2009 & 400 & 368 & 8.0 & Overall \% patients readmitted & @ 12 months & $59.1(110 / 186)$ & $58.2(106 / 182)$ & $>0.05$ \\
\hline
\end{tabular}




\begin{tabular}{|c|c|c|c|c|c|c|c|c|}
\hline Author, date & 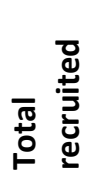 & 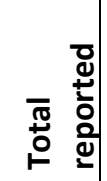 & 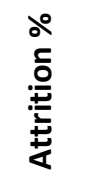 & Indicator description & Timing & $\begin{array}{l}\text { Control } \\
\text { estimate } \%(n / N)\end{array}$ & $\begin{array}{l}\text { Intervention } \\
\text { estimate \% } \\
(n / N)\end{array}$ & $\begin{array}{c}\text { Estimate of } \\
\text { Difference } \\
\text { and/or } p \text { value }\end{array}$ \\
\hline Gillespie,[40] 2009 & 400 & 368 & 8.0 & Overall \% drug-related readmissions & @ 12 months & $24.0(45 / 186)$ & $4.9(9 / 182)$ & $<0.05$ \\
\hline $\begin{array}{l}\text { Komagamine,[43] } \\
2017\end{array}$ & 164 & 158 & 3.7 & Unplanned hospital admission & @ 8 months & $18.9(25 / 132)$ & $21.9(7 / 32)$ & 0.80 \\
\hline Curtin,[42] 2020 & 130 & 130 & 0 & Unplanned hospital admission & @ 3 months & $8.0(5 / 65)$ & $14.0(9 / 65)$ & 0.27 \\
\hline Gillespie,[40] 2009 & 400 & 368 & 8.0 & Hospital ED visits & @ 12 months & $50.0(93 / 186)$ & $26.9(49 / 182)$ & $<0.05$ \\
\hline Curtin,[42] 2020 & 130 & 130 & 0 & Hospital ED visits & @ 3 months & $8.0(5 / 65)$ & $5.0(3 / 65)$ & 0.72 \\
\hline Crotty,[39] 2004 & 110 & 88 & 20.0 & Hospital ED visit or readmission ${ }^{b}$ & @ 2 months & $29.5(13 / 44)$ & $11.4(5 / 44)$ & 0.035 \\
\hline \multicolumn{9}{|l|}{ Other clinical } \\
\hline Gillespie,[40] 2009 & 400 & 368 & 8.0 & Death & @12 months & $32.8(61 / 186)$ & $31.3(57 / 182)$ & 0.82 \\
\hline $\begin{array}{l}\text { Komagamine, [43] } \\
2017\end{array}$ & 164 & 158 & 3.7 & Death & @ 8 months & $9.8(13 / 132)$ & $12.5(4 / 32)$ & 0.75 \\
\hline Curtin,[42] 2020 & 130 & 130 & 0 & Death & @ 3 months & $28.0(18 / 65)$ & $18.90(12 / 65)$ & 0.22 \\
\hline Kutner, [36] 2015 & 381 & 371 & 2.6 & Death & @ 2 months & $20.3(39 / 192)$ & $23.8(45 / 189)$ & 0.36 \\
\hline
\end{tabular}

PIM+ potentially inappropriate medication

a as reported

${ }^{b}$ combined estimate

$R R=$ risk ratio

$n / N=$ number with the outcome over the total number in the intervention or control group 


\section{LIST OF SUPPLEMENTARY FILES}

Supplement 1, Table S1. CriSTAL criteria

Supplement 1, Table 2, Search Strategy by source database

Supplement 1, Table 1.3 Reasons for exclusion after full text assessment

Supplement 2, Table S2.1 Risk of bias key questions

Supplement 2, Figure 2.1 Risk of bias summaries

Supplement 2, Table S2.2 Intervention descriptions 